\title{
Various Modalities Accurate in Diagnosing a Disrupted or Disconnected Pancreatic Duct in Acute Pancreatitis: A Systematic Review
}

\author{
Hester C. Timmerhuis ${ }^{1,2}{ }^{(1)}$. Sven M. van Dijk ${ }^{1,3} \cdot$ Robert C. Verdonk $^{4} \cdot$ Thomas L. Bollen $^{5} \cdot$ Marco J. Bruno $^{6}$. \\ Paul Fockens ${ }^{7} \cdot$ Jeanin E. van Hooft ${ }^{7} \cdot$ Rogier P. Voermans $^{7} \cdot$ Marc G. Besselink $^{3} \cdot$ Hjalmar C. van Santvoort $^{2,8} \cdot$ for the $^{2}$ \\ Dutch Pancreatitis Study Group
}

Received: 13 January 2020 / Accepted: 14 June 2020 / Published online: 27 June 2020

(c) The Author(s) 2020

\begin{abstract}
Background Severe pancreatitis may result in a disrupted pancreatic duct, which is associated with a complicated clinical course. Diagnosis of a disrupted pancreatic duct is not standardized in clinical practice or international guidelines. We performed a systematic review of the literature on imaging modalities for diagnosing a disrupted pancreatic duct in patients with acute pancreatitis.

Methods A systematic search was performed in PubMed, Embase and Cochrane library databases to identify all studies evaluating diagnostic modalities for the diagnosis of a disrupted pancreatic duct in acute pancreatitis. All data regarding diagnostic accuracy were extracted.

Results We included 8 studies, evaluating five different diagnostic modalities in 142 patients with severe acute pancreatitis. Study quality was assessed, with proportionally divided high and low risk of bias and low applicability concerns in $75 \%$ of the studies. A sensitivity of $100 \%$ was reported for endoscopic ultrasound and endoscopic retrograde cholangiopancreatography. The sensitivity of magnetic resonance cholangiopancreatography with or without secretin was $83 \%$. A sensitivity of $92 \%$ was demonstrated for a combined cohort of secretin-magnetic resonance cholangiopancreatography and magnetic resonance cholangiopancreatography. A sensitivity of $100 \%$ and specificity of $50 \%$ was found for amylase measurements in drain fluid compared with ERCP.

Conclusions This review suggests that various diagnostic modalities are accurate in diagnosing a disrupted pancreatic duct in patients with acute pancreatitis. Amylase measurement in drain fluid should be standardized. Given the invasive nature of other modalities, secretin-magnetic resonance cholangiopancreatography or magnetic resonance cholangiopancreatography would be recommended as first diagnostic modality. Further prospective studies, however, are needed.
\end{abstract}

Keywords Acute necrotizing pancreatitis $\cdot$ Pancreatic fistula $\cdot$ Disrupted pancreatic duct $\cdot$ Disconnected pancreatic duct

Presented at the 13th E-AHPBA congress, Amsterdam on June 5th, 2019. Poster presentation at the 51st EPC congress, Bergen on June 28th, 2019. Poster presentation at the 27th UEG Week, Barcelona on October 23, 2019.

Electronic supplementary material The online version of this article (https://doi.org/10.1007/s10620-020-06413-0) contains supplementary material, which is available to authorized users.

Hjalmar C. van Santvoort

h.vansantvoort@umcutrecht.nl

Extended author information available on the last page of the article

\section{Introduction}

Acute pancreatitis is one of the most common gastrointestinal diseases for acute hospital admission [1-3]. The disease course is generally mild. Around $20 \%$ of patients, however, develop necrosis of the pancreatic or peripancreatic tissue [4-7]. This necrosis of the pancreatic parenchyma results in loss of viable pancreatic tissue and potentially loss of integrity of the pancreatic duct [8]. This may cause either a disruption or disconnection of the pancreatic duct, causing leakage of pancreatic fluids in the surrounding tissue or to other organs. A complicated course often follows, which may be characterized by recurrent 
or persistent peripancreatic fluid collections, pancreatic ascites, or pancreatic fistula including external fistulas following percutaneous catheter drainage [9-15]. This causes a major burden on the patient's quality of life and is associated with high healthcare resource utilization [8, 16-18].

The diagnosis of a disrupted or disconnected pancreatic duct syndrome is not standardized [19, 20]. Diagnostic modalities currently used are computed tomography (CT), endoscopic retrograde cholangiopancreatography (ERCP), endoscopic ultrasound (EUS), magnetic resonance cholangiopancreatography (MRCP) with or without secretin, or pancreatography during surgery $[8,13,17,21-29]$. Nowadays, ERCP is still considered as the reference of standard, but this is an invasive procedure with a risk of complications such as secondary infection of pancreatic necrosis, flare of pancreatitis, bleeding and perforation [30, 31].

Because treatment success is related to the degree and location of the disruption [28, 32, 33], a timely and accurate diagnosis of a pancreatic duct disconnection and disruption is expected to facilitate treatment decisions. Evidence-based guidelines are variable regarding the preferred method and timing of diagnosing a disrupted or disconnected pancreatic duct syndrome in acute necrotizing pancreatitis and clear guidelines are missing [19, 20, $34,35]$.

We performed a systematic review to determine the accuracy of the various diagnostic modalities to assess a pancreatic duct disruption and disconnection in patients with severe acute pancreatitis.

\section{Methods}

\section{Search and Study Selection}

This systematic review was conducted according to the Preferred Reporting Items for Systematic reviews and Meta-Analyses (PRISMA) guidelines [36]. A systematic search was performed in PubMed, Embase and Cochrane Library from database inception until April 28, 2020. The Embase search was limited to Embase sources and restricted to publication types (inclusion of articles and systematic reviews). Grey literature (i.e., conference abstracts, editorials and dissertations) was excluded. Search terms included severe pancreatitis (study population), disconnected duct, pancreatic fistula (outcome) and all synonyms. A manual cross-reference check was performed on all studies reviewed during full-text article assessment. Detailed search details are presented in Appendix Table 1-3. The review protocol was not registered online (e.g., PROSPERO) but is available upon request.

\section{Eligibility Criteria}

Eligibility assessment was independently performed by two reviewers (HCT, SMvD) in a standardized manner. Discrepancies were resolved by consensus, after discussion in a meeting of the Dutch Pancreatitis Study Group. After removal of duplicates, the remaining articles were screened on relevance by title/abstract. Reviews, letters, case reports and book chapters were excluded. Selection was restricted to Dutch, German or English human studies with full-text availability. No other restrictions were imposed. Subsequently, full text was assessed for eligibility. Studies were considered eligible if they were cross-sectional studies, cohort studies (with a minimum number of 5 patients) or randomized trials including patients over 18 years of age with acute pancreatitis who underwent any diagnostic modality for a suspected disrupted or disconnected pancreatic duct.

Distinction between a disrupted (partial) and disconnected (complete) pancreatic duct disruption was considered but was not deemed possible owing to heterogeneous index test and the limited number of studies covering this subject.

\section{Data Extraction and Critical Appraisal}

Relevant study characteristics were extracted using a data extraction template based on the Standards of Reporting of Diagnostic Accuracy (STARD) studies checklist [37]. The following information was extracted: (1) country of origin, year of publication, design, setting, inclusion criteria; (2) the performed index test and reference standards; (3) total number of patients included and number of relevant patients; (4) accuracy measurements calculated by constructing $2 \times 2$ tables derived from each index test and its corresponding reference standard.

Data were extracted regarding the imaging characteristics: type of imaging modality, (quality) scoring criteria, data regarding diagnostic accuracy, technical features for each modality and reported observer experience.

The methodological quality of included study was assessed by two reviewers independently (HCT, SMvD) using the Quality Assessment of Diagnostic Accuracy Studies-2 (QUADAS-2) tool [38]. Differences in assessment were resolved by consensus between the two reviewers, or after discussion in a meeting of the Dutch Pancreatitis Study Group.

\section{Statistical Analysis}

For each included study, a $2 \times 2$ contingency table was constructed for each imaging modality. If data were available, sensitivity, specificity, and overall accuracy were calculated 
from the reconstructed tables. Pooled estimates were considered but were assumed trivial owing to heterogeneous index tests and the limited number of studies covering this subject.

\section{Results}

\section{Search}

The initial search yielded 4059 articles; 1565 articles returned from MEDLINE, 1718 from Embase, and 776 results from Cochrane library. After removal of duplicates, 2945 articles remained. Based on title and abstract screening, 76 articles remained for full-text review. Full-text assessment excluded 68 articles. No additional articles were identified after cross-reference check. Four articles evaluated diagnostic modalities; however, they did not provide sufficient data to reconstruct $2 \times 2$ tables and calculate the diagnostic accuracy values or even the sensitivity or specificity and were therefore excluded [11, 39-41]. Finally, 8 articles met the predetermined eligibility criteria [13, 21-25, 27, 28] (Fig. 1, Flowchart). The excluded-by-reason articles are reported in Appendix Table 2.

\section{Study Characteristics}

Study characteristics, including the reference standard for the diagnosis of a disrupted or disconnected pancreatic duct syndrome, are presented in Table 1. Data extracted regarding the imaging characteristics are presented in Appendix Table 3.

The 8 included studies were observational cohort studies published between 2003 and 2016. Two studies collected data in a prospective manner [22, 24]; Gillams et al. did not report the study design [27]. A total of 237 patients with moderate to severe acute pancreatitis, according the revised 2012 Atlanta classification, were included in the studies [4]. In 199 of the 271 patients, (peri)pancreatic necrosis was reported [13, 21, 24, 25, 28]. The number of relevant patients included in the study ranged from 6 to 31, with a total of 142 relevant patients. Four studies primarily investigated the diagnostic accuracy of an imaging modality [23, $24,27]$, three studies primarily investigated the best therapy for a disrupted or disconnected pancreatic duct syndrome $[13,21,22,25]$, and in the last study both were performed [28].

The studies concerning amylase measurements in drain fluid compared their index test with ERCP as reference
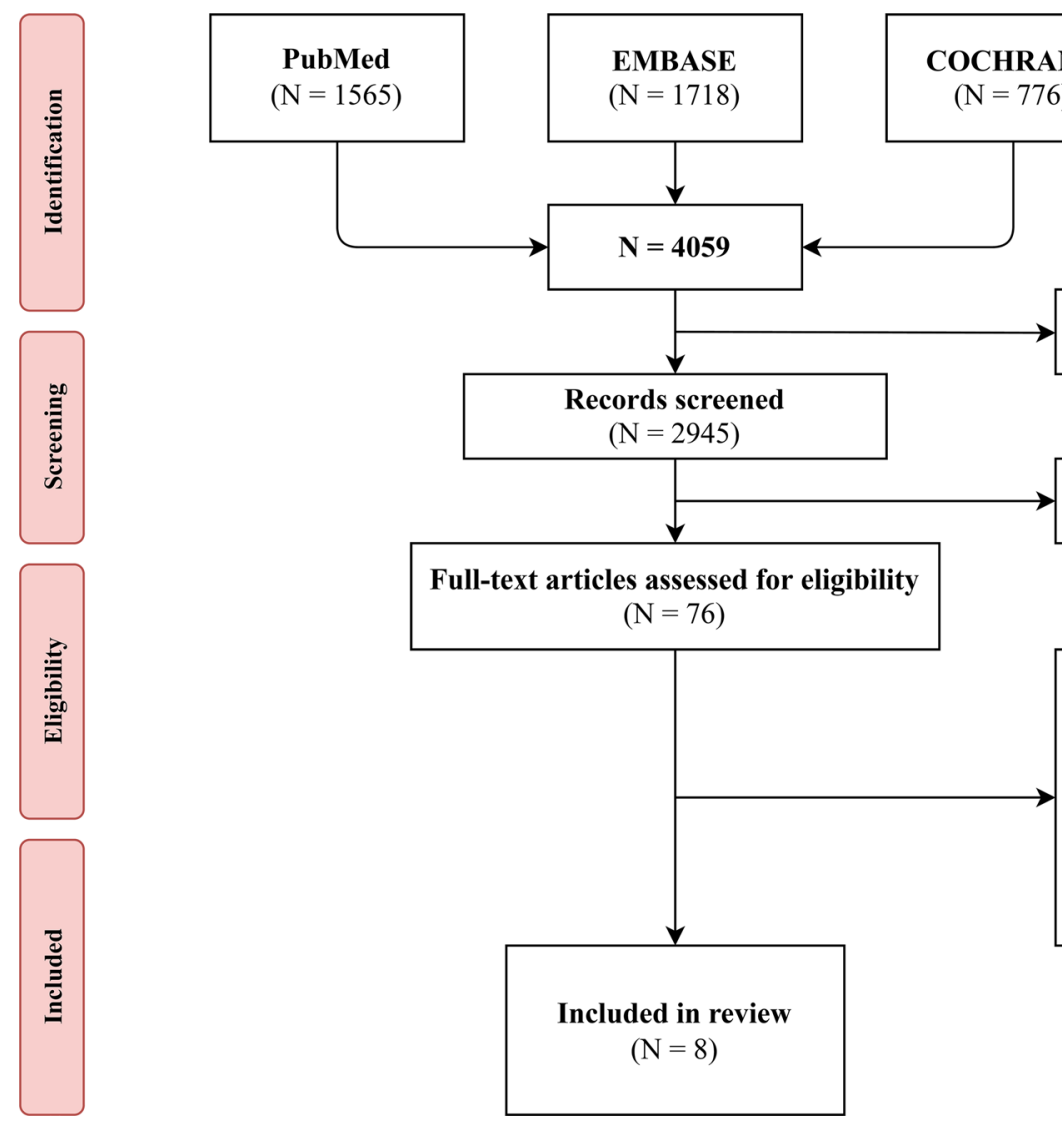

Records excluded based on title and abstract $(\mathrm{N}=2869)$

$\quad$ Full-text articles excluded
\[ (\mathrm{N}=64) \]
No data regarding diagnostics $(\mathrm{N}=39)$
Study design (review, case-report, editorial) $(\mathrm{N}=13)$
No full text available $(\mathrm{N}=5)$
No reference standard $(\mathrm{N}=4)$
Other etiology $(\mathrm{N}=4)$
Less than 5 relevant patients $(\mathrm{N}=1)$

Fig. 1 Flowchart 


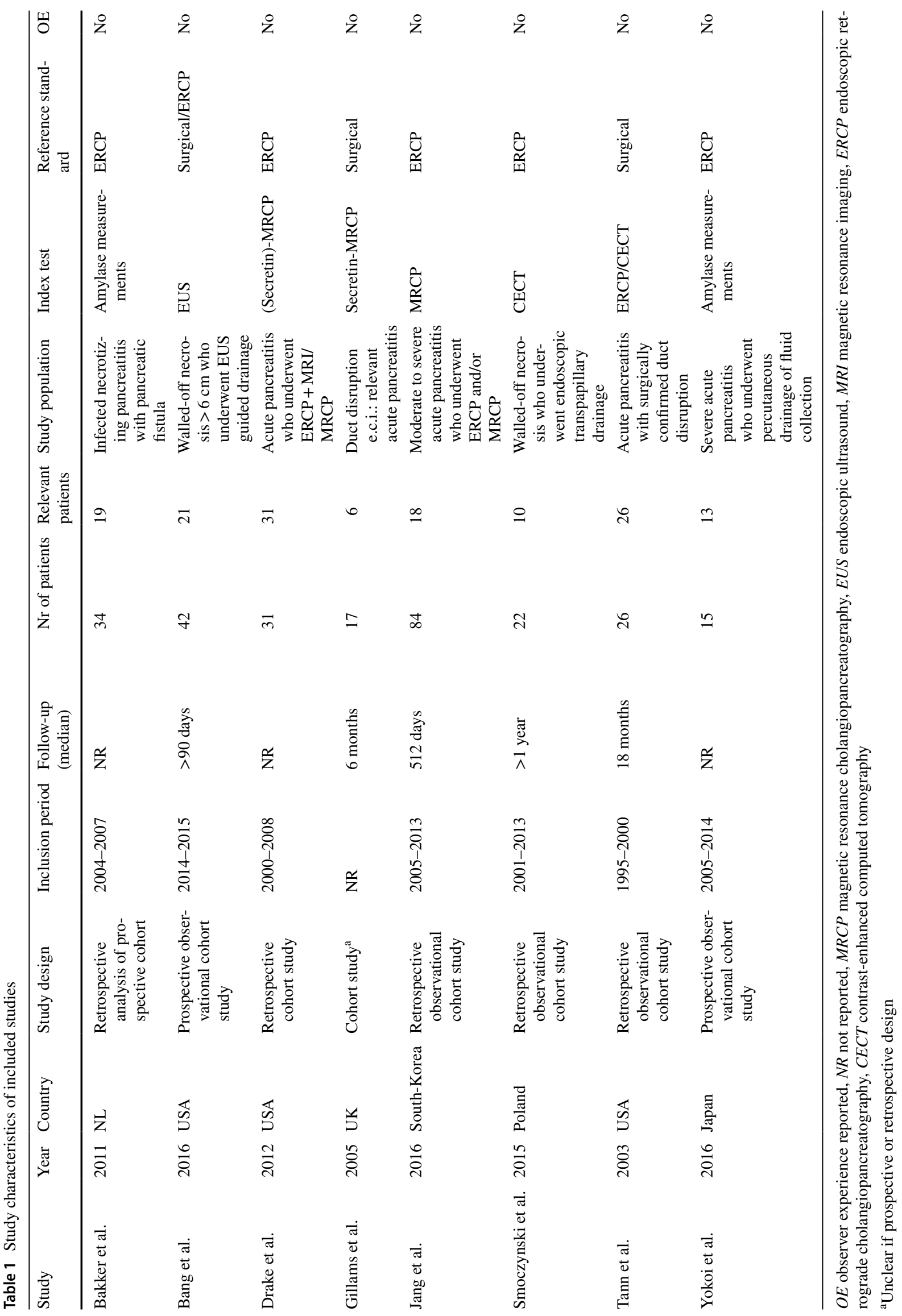


standard [21, 25]. Tann et al. compared ERCP with surgical confirmation [13] and in the study by Bang et al. EUS was compared with either ERCP or surgical confirmation [24]. Contrast-enhanced CT was evaluated in two studies, as compared with ERCP by Smoczynski et al. [21] or surgical confirmation by Tann et al. [13]. Three studies compared either MRCP, secretin-MRCP, or both, with ERCP or surgical confirmation as reference standard. Gillams et al. investigated diagnostic accuracy of secretin-MRCP with surgical confirmation as reference standard [27], comparison of standard MRCP, with ERCP as reference standard, was done in the study by Jang et al. [28], and in the study of Drake et al., no distinction could be made between the patients who underwent standard MRCP or secretin-MRCP, as compared with ERCP [23].

\section{Quality Assessment}

The QUADAS-2 assessment for each domain is depicted in Fig. 2. [Figure 2, Summary of study quality (QUADAS-2)].
An outline of each individual study is presented in Appendix Table 4 . The risk of bias was divided proportionally with a low and high risk of bias in 50\% of the studies. Applicability concerns were low in $75 \%$, high in $21 \%$ and unclear in $4 \%$ of the studies.

\section{Diagnostic Accuracy}

Results on diagnostic accuracy of the different imaging modalities studies are summarized in Table 2. Additional findings are presented in Appendix Table 5.

\section{Amylase Measurement in Drain Fluid}

Yokoi et al. and Bakker et al. investigated the clinical value of amylase measurements in drain fluid in detecting pancreatic fistula and a disrupted pancreatic duct in patients with severe acute pancreatitis who underwent percutaneous drainage of a fluid collection. The presence of pancreatic fistula was, in both studies, defined as output of drain fluid

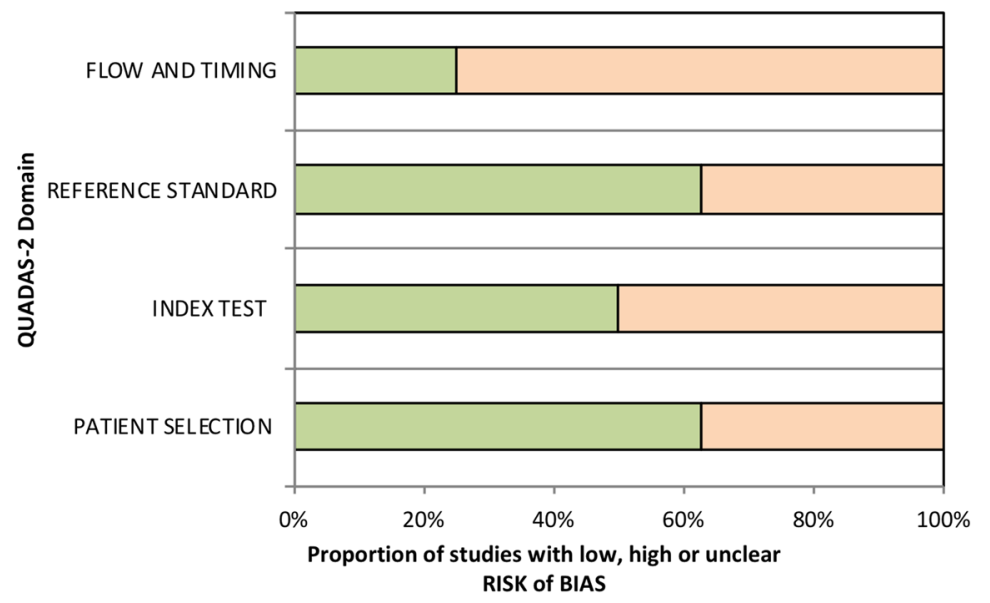

口Low $\square$ High $\square$ Unclear

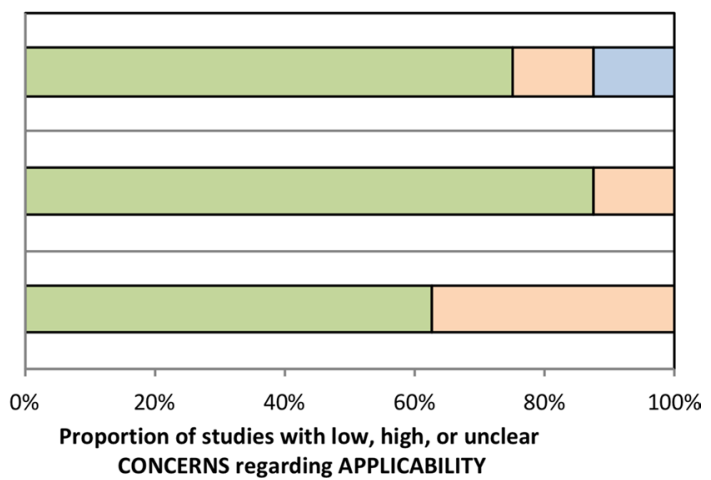

Fig. 2 Summary of study quality (QUADAS-2)

Table 2 Results of included studies

\begin{tabular}{|c|c|c|c|c|c|c|c|c|c|c|}
\hline Study & Index test & Reference standard & $\begin{array}{l}\text { Relevant } \\
\text { patients }\end{array}$ & $\mathrm{TP}$ & FN & $\mathrm{FP}$ & $\mathrm{TN}$ & Sensitivity (\%) & Specificity & Overall accuracy \\
\hline Bakker et al. & Amylase measurements & $\mathrm{ERCP}$ & 19 & 18 & 0 & 1 & 0 & 100 & & \\
\hline \multirow[t]{2}{*}{ Bang et al. } & EUS & Surgical & 21 & 1 & 0 & - & - & 100 & - & - \\
\hline & EUS & ERCP & & 20 & 0 & - & - & 100 & - & - \\
\hline Drake et al. & (secretin)-MRCP & ERCP & 31 & 23 & 2 & 0 & 8 & 92 & $100 \%$ & $94 \%$ \\
\hline Gillams et al. & Secretin-MRCP & Surgical & 6 & 5 & 1 & - & - & 83.3 & - & - \\
\hline Jang et al. & MRCP & ERCP & 18 & 15 & 3 & 0 & 3 & 83.3 & $100 \%$ & $85.7 \%$ \\
\hline Smoczynski et al. & CT & ERCP & 10 & 8 & 2 & - & - & 80 & - & - \\
\hline \multirow[t]{2}{*}{ Tann et al. } & ERCP & Surgical & 26 & 26 & 0 & - & - & 100 & - & - \\
\hline & $\mathrm{CT}$ & Surgical & & 26 & 26 & & & 0 & & \\
\hline Yokoi et al. & Amylase measurements & ERCP & 13 & 6 & 0 & 7 & 7 & 100 & $50 \%$ & $65 \%$ \\
\hline
\end{tabular}


with an amylase content of more than three times the normal serum amylase level. A comparison was made between amylase measurement and ERCP, with a similar sensitivity found of $100 \%$. A specificity of $50 \%$, resulting in an overall diagnostic accuracy of $65 \%$ for amylase measurements in drain fluid, was found by Yokoi et al. [22, 25].

\section{ERCP}

Tann et al. evaluated the diagnostic accuracy of ERCP. A disrupted or disconnected pancreatic duct was confirmed during surgical correction of a disrupted pancreatic duct with a sensitivity of $100 \%$. No data could be extracted to calculate specificity. This study also evaluated prior CTscans of patients diagnosed with disrupted or disconnected pancreatic duct syndrome by ERCP and none of the prior CT interpretation correctly identified a disrupted or disconnected pancreatic duct [13].

\section{Endoscopic Ultrasound}

A sensitivity of $100 \%$ for detecting a disrupted pancreatic duct with EUS was found in patients with a walled-off necrosis of $>6 \mathrm{~cm}$, in whom the pancreatic duct was visible during EUS, as confirmed with histopathological confirmation after distal pancreatectomy or ERCP in the study by Bang et al. [24]. No specificity was reported or could be calculated.

\section{Contrast-Enhanced CT}

A sensitivity of $80 \%$ was found by Smoczynski et al. comparing contrast-enhanced CT with ERCP in patients with walled-off necrosis who underwent endoscopic transpapillary drainage [21]. Tann et al. compared contrast-enhanced CT with surgical confirmation during surgical correction for a disrupted pancreatic duct in patients with moderate to severe pancreatitis. A sensitivity of $0 \%$ was found [13]. For both studies, no specificity was reported or could be calculated from the extracted data.

\section{MRCP}

Jang et al. investigated the diagnostic yield of MRCP (without secretin) for detecting a pancreatic duct disruption, with ERCP as reference standard [28] in patients with moderate to severe pancreatitis. A sensitivity of $100 \%$ was reported. No specificity was reported and could not be calculated from the extracted data.

\section{Secretin-MRCP}

Secretin-MRCP, as compared with surgical confirmation of a disrupted pancreatic duct, was evaluated by Gillams et al. in patients with moderate to severe pancreatitis with a reported sensitivity of $83.3 \%$. Again, specificity was not reported or could not be calculated from the source data [27].

\section{MRCP and Secretin-MRCP}

In the study by Jang et al., both secretin-MRCP and MRCP were compared with ERCP in patients with moderate to severe pancreatitis, showing a sensitivity of $92 \%$, a specificity of $100 \%$, and an overall accuracy of $94 \%$. The diagnostic difference between secretin-MRCP and MRCP was not reported upon [23].

\section{Discussion}

This is the first systematic review evaluating the various diagnostic modalities for diagnosing a disrupted pancreatic duct in moderate to severe acute pancreatitis. A sensitivity of $100 \%$ was demonstrated for amylase measurements in drain fluid and ERCP [13, 17, 22, 25]. The sensitivity for MRCP and for secretin-MRCP was $83 \%$, both with a specificity of $100 \%$ [27, 28]. For a combined cohort of MRCP and secretin-MRCP a sensitivity of $92 \%$ was found [23]. Abdominal contrast-enhanced CT had the lowest sensitivity, ranging from 0 to $80 \%[13,21]$.

In clinical practice, a suspicion on a disrupted or disconnected pancreatic duct is raised if amylase content in drain fluid contains more than three times the normal serum amylase level [13, 17, 22, 25, 42, 43]. Amylase measurement in drain fluid, however, does not distinguish between a partial and complete disruption. To confirm this suspicion and the degree of disruption, the current reference standard to diagnose a disrupted or disconnected pancreatic duct is ERCP. This imaging modality is, however, invasive and increases the risk of infected necrosis and other complications in this patient population [30, 31]. These risks do not occur with EUS or less invasive imaging modalities such as (secretin)-MRCP.

Studies on the accuracy of MRCP or EUS for detecting a disrupted duct in acute pancreatitis are scarce. Only Bang et al. evaluated EUS, which demonstrated a sensitivity of $100 \%$. Notably, this study only included patients with a walled-off necrosis of more than $6 \mathrm{~cm}$ and a disrupted duct was present in $95 \%$ of cases. Moreover, 9 patients were excluded because EUS characterization of the pancreatic upstream gland was suboptimal. If this had been included, the sensitivity would have been considerably lower. Success of visualization was dependent on the size of the walled-off necrosis, which raises the suggestion that EUS is only of added value in a selective patient group [24]. Furthermore, complete disconnection may be seen on EUS, but visualization of a partial disruption is not possible on EUS. In this 
review, we included two studies that used secretin during MRCP [23, 27]. One study evaluating secretin-MRCP was excluded due to the limited number of relevant patients $(n=3)$; this study reported a sensitivity of $100 \%$ for secretin-MRCP compared with ERCP [26]. Secretin-MRCP or MRCP can be performed in nearly all patients [26]. Secretin is added for stimulation of pancreatic secretions to facilitate the identification of the pancreatic duct. No complications were reported after administration of secretin [26, 27]. In one study, only 6 of 31 patients received secretin and in 2 patients, who did not receive secretin, a disruption of the pancreatic duct was missed on MRCP. This raises the question of this may have been avoided with the use of secretin [23]. Even though the reported visualization of the pancreatic duct is generally very good, one study reported a poorer visualization by MRCP, as compared with ERCP, in patients with a partial disrupted pancreatic duct, as compared with patients with a completely disrupted pancreatic duct [28]. No secretin was used in this study, which may explain the poor visualization of the pancreatic duct. Another advantage of secretin-MRCP, besides accurate visualization of the pancreatic duct for detection of a disruption or disconnection, is an accurate visualization of the biliary duct. This may aid in establishing the cause of pancreatitis or evaluation of complications such as biliary obstruction due to peripancreatic collections or inflammation [26, 44].

Besides lack of clear evidence and guidelines for the diagnosis, there is also no consensus on the optimal treatment strategy of disrupted or disconnected duct. Several studies have reported high success rates of various treatment strategies including percutaneous drainage, endoscopic drainage and surgical resection $[8,11,13,14,16,18,21-24$, $26,28,29,32,33,39-41,45-60]$. Yet, many of these studies were retrospective studies comprising selected patient cohorts. There are no large prospective studies of consecutive patients with necrotizing pancreatitis, specifically evaluating the prevalence or treatment outcome of a disrupted or disconnected pancreatic duct. The success rate of conservative treatment also is unknown [61]. Future studies should investigate the optimal timing, method and sequence of invasive interventions in this group of patients. To design these studies, a better understanding of the natural course of a disrupted or disconnected pancreatic duct in several subgroups of patients is needed. For instance, as success rates of different treatment strategies are probably related to the degree and location of the disruption, a distinction must be made between a partial and complete disruption. A partial disruption can often be bridged with a pancreatic duct stent, while it is very difficult to bridge a complete disruption [28, $32,33]$. Therefore, a timely and accurate diagnosis of a pancreatic duct disruption and disconnection will provide better possibilities to predict which treatment could be successful in a specific patient. There are currently no broadly accepted definitions on a partial disrupted, a complete disrupted, a disconnected or disconnected gland syndrome [13]. Subsequently, the diagnostic criteria for a partial disrupted or complete disrupted or disconnected pancreatic duct syndrome varied in the studies included in this review. The distinction between a disrupted (partial) and disconnected (complete) pancreatic duct disruption in this systematic review was considered but was not possible owing to heterogeneous index tests and the limited number of studies covering this subject. Therefore, for this systematic review we have consciously chosen to focus on disruption and disconnection together to outline the different diagnostic modalities used to diagnose either disrupted or disconnected pancreatic duct. An important concern, diagnostic accuracy of a modality for a partial disruption may differ from the diagnostic accuracy for a complete disconnection. This difference could not be made on the extracted date and literature in this systematic review.

This study had some limitations. First, only a few studies could be included and hence number of eligible patients was relatively low. This should be taken into account when considering the calculated sensitivity. Second, most studies were performed in selected patient cohorts and were not designed to answer this question and suffered from high risk of bias. Subsequently, no clear pre-specified index test and clearly defined reference standard were used. Third, partial verification bias was present in some of the included studies [22, 23, $28]$ and the flow and timing of these tests may impose bias. Also, no standardized definitions for a disrupted pancreatic duct were used. As last, in four studies no specificity could be calculated [13, 21, 24, 27]. We have not registered our study in the PROSPERO database; however, our study protocol was prospectively designed.

This study is the only study comparing different diagnostic modalities in diagnosing a disrupted or disconnected pancreatic duct. Strengths of this review included the use of exhaustive search technique (in the major databases with small restrictions to publication type and grey literature) by two reviewers independently and validated systematic review methods, which strengthens our conclusions.

In conclusion, this systematic review suggests that EUS, ERCP, MRCP and secretin-MRCP appear all accurate in diagnosing a disruption or disconnection of the pancreatic duct in patients with acute pancreatitis. Amylase measurements in drain fluid should be standardized after percutaneous catheter drainage or surgical drain placement. Given the poor overall visualization of the pancreatic duct in a substantial number of patients with necrotizing pancreatitis on EUS and CT and the invasive nature of ERCP, MRCP or secretin-MRCP is recommended as first diagnostic modality. These results, however, should be taken with caution due to poor methodological quality of included studies and small sample sizes. Further prospective studies are needed to define the optimal timing and the accurate diagnostic value 
of (secretin-)MRCP in different subgroups of patients with necrotizing pancreatitis.

Funding The execution of this review was funded by St. Antonius Hospital Research Fund.

\section{Compliance with Ethical Standards}

Conflict of interest The authors declare that they have no conflict of interest.

Open Access This article is licensed under a Creative Commons Attribution-NonCommercial 4.0 International License, which permits any non-commercial use, sharing, adaptation, distribution and reproduction in any medium or format, as long as you give appropriate credit to the original author(s) and the source, provide a link to the Creative Commons licence, and indicate if changes were made. The images or other third party material in this article are included in the article's Creative Commons licence, unless indicated otherwise in a credit line to the material. If material is not included in the article's Creative Commons licence and your intended use is not permitted by statutory regulation or exceeds the permitted use, you will need to obtain permission directly from the copyright holder. To view a copy of this licence, visit http://creativecommons.org/licenses/by-nc/4.0/.

\section{References}

1. Health \& Social Care Information Centre. Hospital episode statistics admitted patient care-E. https://digital.nhs.uk/data-and-infor mation/publications/statistical/hospital-admitted-patient-careactivity/hospital-episode-statistics-admitted-patient-care-engla nd-2014-15. Accessed April 28, 2020.

2. Van Dijk SM, Hallensleben NDL, Van Santvoort HC, et al. Acute pancreatitis: recent advances through randomised trials. Gut. 2017;66:2024-2032.

3. Besselink MG, van Santvoort HC, Buskens E, et al. Probiotic prophylaxis in predicted severe acute pancreatitis: a randomised, double-blind, placebo-controlled trial. Lancet Lond Engl. 2008;371:651-659.

4. Banks PA, Bollen TL, Dervenis C, et al. Classification of acute pancreatitis-2012: revision of the Atlanta classification and definitions by international consensus. Gut. 2013;62:102-111.

5. Bakker OJ, Van Santvoort H, Besselink MGH, et al. Extrapancreatic necrosis without pancreatic parenchymal necrosis: a separate entity in necrotising pancreatitis? Gut. 2013;62:1475-1480.

6. Párniczky A, Kui B, Szentesi A, et al. Prospective, multicentre, nationwide clinical data from 600 cases of acute pancreatitis. PLoS One. 2016;11:1-19.

7. Van Santvoort HC, Bakker OJ, Bollen TL, et al. A conservative and minimally invasive approach to necrotizing pancreatitis improves outcome. Gastroenterology. 2011;141:1254-1263.

8. Fischer TD, Gutman DS, Hughes SJ, Trevino JG, Behrns KE. Disconnected pancreatic duct syndrome: disease classification and management strategies. J Am Coll Surg. 2014;219:704-712.

9. Arvanitakis M, Delhaye M, Bali M, Matos C, Le Moine O, Deviere J. Endoscopic treatment of external pancreatic fistulas. Gastroenterol Pol. 2007;14:191-195.

10. Larsen M, Kozarek RA. Management of disconnected pancreatic duct syndrome. Curr Treat Options Gastroenterol. 2016;14:348-359.
11. Kamal A, Singh VK, Akshintala VS, et al. CT and MRI assessment of symptomatic organized pancreatic fluid collections and pancreatic duct disruption: an interreader variability study using the revised Atlanta classification 2012. Abdom Imaging. 2015;40:1608-1616.

12. Fulcher AS, Turner MA, Yelon JA, et al. Magnetic resonance cholangiopancreatography (MRCP) in the assessment of pancreatic duct trauma and its sequelae: preliminary findings. $J$ Trauma. 2000;48:1001-1007.

13. Tann M, Maglinte D, Howard TJ, et al. Disconnected pancreatic duct syndrome: imaging findings and therapeutic implications in 26 surgically corrected patients. J Comput Assist Tomogr. 2003;27:577-582.

14. Irani S, Gluck M, Ross A, et al. Resolving external pancreatic fistulas in patients with disconnected pancreatic duct syndrome: using rendezvous techniques to avoid surgery (with video). Gastrointest Endosc. 2012;76:583-586.

15. Rana SS, Sharma R, Kang M, Gupta R. Natural course of low output external pancreatic fistula in patients with disconnected pancreatic duct syndrome following acute necrotising pancreatitis. Pancreatology. 2020;20:177-181.

16. Lawrence C, Howell DA, Stefan AM, et al. Disconnected pancreatic tail syndrome: potential for endoscopic therapy and results of long-term follow-up. Gastrointest Endosc. 2008;67:673-679.

17. Nadkarni NA, Kotwal V, Sarr MG, Vege SS. Disconnected pancreatic duct syndrome: endoscopic stent or surgeon's knife? Pancreas. 2015;44:16-22.

18. Pelaez-Luna M, Vege SS, Petersen BT, et al. Disconnected pancreatic duct syndrome in severe acute pancreatitis: clinical and imaging characteristics and outcomes in a cohort of 31 cases. Gastrointest Endosc. 2008;68:91-97.

19. Group W, Apa IAP, Pancreatitis A. IAP/APA evidence-based guidelines for the management of acute pancreatitis. Pancreatology. 2013;13:e1-e15.

20. Arvanitakis M, Dumonceau J-M, Albert J, et al. Endoscopic management of acute necrotizing pancreatitis: European society of gastrointestinal endoscopy (ESGE) evidence-based multidisciplinary guidelines. Endoscopy. 2018;50:524-546.

21. Smoczyński M, Jagielski M, Jabłońska A, Adrych K. Transpapillary drainage of walled-off pancreatic necrosis-a single center experience. Videosurg Other Miniinvasive Tech/Wideochirurgia i Inne Tech Malo Inwazyjne. 2015;10:527-533.

22. Yokoi Y, Kikuyama M, Kurokami T, Sato T, et al. Early dual drainage combining transpapillary endotherapy and percutaneous catheter drainage in patients with pancreatic fistula associated with severe acute pancreatitis. Pancreatology. 2016;16:497-507.

23. Drake LM, Anis M, Lawrence C. Accuracy of magnetic resonance cholangiopancreatography in identifying pancreatic duct disruption. J Clin Gastroenterol. 2012;46:696-699.

24. Bang JY, Navaneethan U, Hasan MK, Hawes RH, Varadarajulu S, et al. EUS correlates of disconnected pancreatic duct syndrome in walled-off necrosis. Endosc Int Open. 2016;4:E883-E889.

25. Bakker OJ, van Baal MC, van Santvoort HC, et al. Endoscopic transpapillary stenting or conservative treatment for pancreatic fistulas in necrotizing pancreatitis: multicenter series and literature review. Ann Surg. 2011;253:961-967.

26. Arvanitakis M, Delhaye M, De Maertelaere V, et al. Computed tomography and magnetic resonance imaging in the assessment of acute pancreatitis. Gastroenterology. 2004;126:715-723.

27. Gillams AR, Kurzawinski T, Lees WR. Diagnosis of duct disruption and assessment of pancreatic leak with dynamic secretinstimulated MR cholangiopancreatography. AJR Am J Roentgenol. 2006;186:499-506. 
28. Jang JW, Kim M-H, Oh D, et al. Factors and outcomes associated with pancreatic duct disruption in patients with acute necrotizing pancreatitis. Pancreatology. 2016;16:958-965.

29. Telford JJ, Farrell JJ, Saltzman JR, et al. Pancreatic stent placement for duct disruption. Gastrointest Endosc. 2002;56:18-24.

30. Woods RW, Akshintala VS, Singh VK, et al. CT severity of postERCP pancreatitis: results from a single tertiary medical center. Abdom Imaging. 2014;39:1162-1168.

31. Karjula H, Nordblad Schmidt P, Makela J, Liisanantti JH, Ohtonen $\mathrm{P}$, Saarela A. Prophylactic pancreatic duct stenting in severe acute necrotizing pancreatitis: a prospective randomized study. Endoscopy. 2019;11:1027-1034.

32. Das R, Papachristou GI, Slivka A, et al. Endotherapy is effective for pancreatic ductal disruption: a dual center experience. Pancreatology. 2016;16:278-283.

33. Rana SS, Bhasin DK, Nanda M, et al. Endoscopic transpapillary drainage for external fistulas developing after surgical or radiological pancreatic interventions. J Gastroenterol Hepatol. 2010;25:1087-1092.

34. Banks PA, Freeman ML, Practice Parameters Committee of the American College of Gastroenterology. Practice guidelines in acute pancreatitis. Am J Gastroenterol. 2006;101:2379-2400.

35. Baron TH, DiMaio CJ, Wang AY, Morgan KA. American gastroenterological association clinical practice update: management of pancreatic necrosis. Gastroenterology. 2020;158:67.e1-75.e1.

36. Moher D, Liberati A, Tetzlaff J, Altman DG. Systematic reviews and meta-analyses: the PRISMA statement. Ann Intern Med. 2009;151:264-269.

37. Cohen JF, Korevaar DA, Altman DG, et al. STARD 2015 guidelines for reporting diagnostic accuracy studies: explanation and elaboration. BMJ Open. 2016;6:e 012799.

38. Whiting PF, Rutjes AWS, Westwood ME, et al. QUADAS-2: a revised tool for the quality assessment of diagnostic accuracy studies. Ann Intern Med. 2011;155:529-536.

39. Uomo G, Molino D, Visconti M, Ragozzino A, Manes G, Rabitti PG. The incidence of main pancreatic duct disruption in severe biliary pancreatitis. Am J Surg. 1998;176:49-52.

40. Lau ST, Simchuk EJ, Kozarek RA, Traverso LW, et al. A pancreatic ductal leak should be sought to direct treatment in patients with acute pancreatitis. Am J Surg. 2001;181:411-415.

41. Peng R, Zhang XM, Ji YF, et al. Pancreatic duct patterns in acute pancreatitis: a MRI study. PLoS One. 2013;8:e72792.

42. Halttunen J, Weckman L, Kemppainen E, Kylanpaa ML. The endoscopic management of pancreatic fistulas. Surg Endosc. 2005;19:559-562.

43. Jin S, Shi X-J, Wang S-Y, et al. Drainage fluid and serum amylase levels accurately predict development of postoperative pancreatic fistula. World J Gastroenterol. 2017;23:6357-6364.

44. Akisik MF, Sandrasegaran K, Aisen AA, Maglinte DDT, Sherman S, Lehman GA. Dynamic secretin-enhanced MR cholangiopancreatography. Radiographics. 2006;26:665-677.

45. Beck WC, Bhutani MS, Raju GS, Nealon WH, et al. Surgical management of late sequelae in survivors of an episode of acute necrotizing pancreatitis. J Am Coll Surg. 2012;214:682-688.

46. Chen Y, Jiang Y, Qian W, et al. Endoscopic transpapillary drainage in disconnected pancreatic duct syndrome after acute pancreatitis and trauma: long-term outcomes in 31 patients. BMC Gastroenterol. 2019;19:54.

47. Deviere J, Bueso H, Baize M, et al. Complete disruption of the main pancreatic duct: endoscopic management. Gastrointest Endosc. 1995;42:445-451.
48. Dua MM, Jensen CW, Friedland S, et al. Isolated pancreatic tail remnants after transgastric necrosectomy can be observed. J Surg Res. 2018;231:109-115.

49. Howard TJ, Rhodes GJ, Selzer DJ, et al. Roux-en-Y internal drainage is the best surgical option to treat patients with disconnected duct syndrome after severe acute pancreatitis. Surgery. 2001;130:714-721.

50. Jagielski M, Smoczynski M, Adrych K. The role of endoscopic treatment of pancreatic duct disruption in patients with walled-off pancreatic necrosis. Surg Endosc. 2018;32:4939-4952.

51. Murage KP, Ball CG, Zyromski NJ, et al. Clinical framework to guide operative decision making in disconnected left pancreatic remnant (DLPR) following acute or chronic pancreatitis. Surgery. 2010;148:847-857.

52. Pearson EG, Scaife CL, Mulvihill SJ, Glasgow RE. Roux-en$Y$ drainage of a pancreatic fistula for disconnected pancreatic duct syndrome after acute necrotizing pancreatitis. $H P B$. 2012;14:26-31.

53. Prakash A, Koshy AK, Rao BH, Venu RP. Endoscopic Ultrasound-Guided Rendezvous Procedure for a Nondilated, Leaking Pancreatic Duct, vol. 5, ACG Case Reports Journal. United States, p. e105; 2018.

54. Rana SS, Sharma RK, Gupta R. Endoscopic management of pancreatic ascites due to duct disruption following acute necrotizing pancreatitis. JGH Open. 2019;3:111-116.

55. Rana SS, Bhasin DK, Sharma R, Gupta R. Factors determining recurrence of fluid collections following migration of intended long term transmural stents in patients with walled off pancreatic necrosis and disconnected pancreatic duct syndrome. Endosc Ultrasound. 2015;4:208-212.

56. Rana SS, Sharma R, Gupta R. Endoscopic treatment of refractory external pancreatic fistulae with disconnected pancreatic duct syndrome. Pancreatology. 2019;19:608-613.

57. Rana SS, Bhasin DK, Rao C, Sharma R, Gupta R. Consequences of long term indwelling transmural stents in patients with walled off pancreatic necrosis and disconnected pancreatic duct syndrome. Pancreatology. 2013;13:486-490.

58. Sharaiha RZ, Tyberg A, Khashab MA, et al. Endoscopic therapy with lumen-apposing metal stents is safe and effective for patients with pancreatic walled-off necrosis. Clin Gastroenterol Hepatol. 2016;14:1797-1803.

59. Téllez-Aviña FI, Casasola-Sánchez LE, Ramírez-Luna MA, et al. Permanent indwelling transmural stents for endoscopic treatment of patients with disconnected pancreatic duct syndrome. J Clin Gastroenterol. 2017;52:85-90.

60. Yamada R, Umeda Y, Shiono Y, et al. Management of the late effects of disconnected pancreatic duct syndrome: a case report. World J Clin Cases. 2019;7:1053-1059.

61. van Dijk SM, Timmerhuis HC, Verdonk RC, et al. Treatment of disrupted and disconnected pancreatic duct in necrotizing pancreatitis: a systematic review and meta-analysis. Pancreatology. 2019;19:905-915.

Publisher's Note Springer Nature remains neutral with regard to jurisdictional claims in published maps and institutional affiliations. 


\section{Affiliations}

\section{Hester C. Timmerhuis ${ }^{1,2}$ - Sven M. van Dijk ${ }^{1,3} \cdot$ Robert C. Verdonk $^{4} \cdot$ Thomas L. Bollen $^{5} \cdot$ Marco J. Bruno $^{6}$. Paul Fockens ${ }^{7} \cdot$ Jeanin E. van Hooft ${ }^{7} \cdot$ Rogier P. Voermans $^{7} \cdot$ Marc G. Besselink $^{3} \cdot$ Hjalmar C. van Santvoort ${ }^{2,8} \cdot$ for the $^{2}$ Dutch Pancreatitis Study Group}

Hester C. Timmerhuis

h.timmerhuis@antoniusziekenhuis.nl

Sven M. van Dijk

s.vandijk@antoniusziekenhuis.nl

Robert C. Verdonk

r.verdonk@antoniusziekenhuis.nl

Thomas L. Bollen

t.bollen@antoniusziekenhuis.nl

Marco J. Bruno

m.bruno@erasmusmc.nl

Paul Fockens

p.fockens@amsterdamumc.nl

Jeanin E. van Hooft

j.e.vanhooft@amsterdamumc.nl

Rogier P. Voermans

r.p.voermans@amsterdamumc.nl

Marc G. Besselink

m.g.besselink@amsterdamumc.nl

1 Department of Research and Development, St. Antonius Hospital, PO 2500, 3430 EM Nieuwegein, The Netherlands
2 Department of Surgery, St. Antonius Hospital, PO 2500, 3430 EM Nieuwegein, The Netherlands

3 Department of Surgery, Amsterdam Gastroenterology and Metabolism, Amsterdam UMC, University of Amsterdam, PO 22660, 1100 DD Amsterdam, The Netherlands

4 Department of Gastroenterology and Hepatology, St. Antonius Hospital, PO 2500, 3430 EM Nieuwegein, The Netherlands

5 Department of Radiology, St. Antonius Hospital, PO 2500, 3430 EM Nieuwegein, The Netherlands

6 Department of Gastroenterology, Erasmus MC University Medical Center, PO 2040, 3000 CA Rotterdam, The Netherlands

7 Department of Gastroenterology and Hepatology, Amsterdam Gastroenterology and Metabolism, Amsterdam UMC, University of Amsterdam, PO 22660, 1100 DD Amsterdam, The Netherlands

8 Department of Surgery, University Medical Center Utrecht, P.O. Box 85500, 3508 GA Utrecht, The Netherlands 